\title{
Modelling turbulent flow from dam break using slow manifolds
}
D. J. Georgiev ${ }^{1}$
A. J. Roberts ${ }^{2}$
D. V. Strunin ${ }^{3}$

(Received 15 August 2008; revised 21 July 2009)

\begin{abstract}
We present a novel approach based on centre manifold techniques to describe the large scale dynamics of the mean turbulent dam-break flow. We avoid empirical assumptions about the cross-stream profile of the velocity; instead a solution is obtained using free surface and bed boundary conditions that accommodate constant turbulent shear as an approximately neutral mode. We describe the turbulent dynamics across the flow, and identify important factors affecting the turbulent dissipation in the lateral direction. Available experimental data verify the results.
\end{abstract}

\section{Contents}

\section{Problem formulation}

\section{C1035}

http://anziamj . austms.org.au/ojs/index.php/ANZIAMJ/article/view/1466 gives this article, (c) Austral. Mathematical Soc. 2009. Published September 3, 2009. ISSN 1446-8735. (Print two pages per sheet of paper.) 
3 Centre manifold technique

C1038

4 Calibration

C1041

5 Dam-break modelling

C1045

6 Conclusion

C1048

References

C1049

\section{Introduction}

Many environmental flows such as rivers, tidal waves and dam-breaks have low aspect ratio of the depth to the lateral extent. Consequently the fluid pressure is close to a hydrostatic balance. Such flows are driven by gravity, which generates developed turbulence and reshapes the free surface. The dam-break flow is an example of such type of flow, where fluid is released from rest following sudden removal of the dam. Dam-break flow has been studied for more than a hundred years, both theoretically [14, e.g.] and experimentally [5, e.g.]. Due to the complexity of the modelling, theoretical models are based upon either a laminar or inviscid formulation, or use various empirical assumptions about the parameters of turbulence. There is a trend to replace these assumptions with direct numerical simulations, large eddy simulations [10, e.g.] or dynamical system approaches [13], as they provide much deeper insight into the dynamics.

We apply modern dynamical systems theory to systematically control error, assess domains of validity, comprehensively account for further physical effects, and resolve internal structures within the dam-break flow. We aim the mathematical analysis to represent the dominant physics. As indicated by Figures $14-15$ by Janosi [5], turbulence induces mixing across the fluid layer; thus, we expect to be able to model the dynamics in terms of depth-averaged quantities. But instead of depth-averaging the equations $[4$, e.g.], we use 
centre manifold theory to resolve turbulent dynamics across the fluid layer and hence provide a sound macroscale closure for the relatively slow, long term, large scale, dynamics of interest to environmental modellers.

\section{Problem formulation}

Flow model Consider 2D flow of an incompressible fluid with constant density, with lateral direction $x_{1}=x$, and normal direction $x_{3}=z$, over a slightly sloping ground, and depth $\eta(x, t)$. The turbulence of the flow is assumed fully developed with turbulent mean velocity field $\vec{u}=(\mathfrak{u}, w)=$ $\left(\mathfrak{u}_{1}, \mathfrak{u}_{3}\right)$. The related turbulent mean strain-rate tensor is

$$
S_{i j}=\frac{1}{2}\left(\frac{\partial u_{i}}{\partial x_{j}}+\frac{\partial u_{j}}{\partial x_{i}}\right)
$$

and its second invariant is

$$
S=\left(\sum_{i, j} S_{i j}^{2}\right)^{1 / 2} .
$$

Mass conservation is described by the continuity equation

$$
\vec{\nabla} \cdot \vec{u}=\frac{\partial u}{\partial x}+\frac{\partial w}{\partial z}=0
$$

and the non-dimensional transport of momentum by

$$
\frac{\partial \vec{u}}{\partial t}+\vec{u} \cdot \vec{\nabla} \vec{u}=-\vec{\nabla} p+\vec{\nabla} \cdot \vec{\tau}+\vec{g},
$$

where $p$ is the turbulent mean pressure field and $\vec{\tau}$ is the turbulent mean deviatoric stress tensor. The forcing of the flow $\vec{g}=\left(g_{1}, g_{3}\right)$ is from gravity. 
Smagorinski turbulence model The effects of turbulence are modelled with the eddy viscosity $v$, which is related to the mean shear stress via the Boussinesq equation

$$
\tau_{i j}=2 v S_{i j} .
$$

We aim to model flows with very large Reynolds number using the Smagorinski model of turbulence [10]. This sets the turbulent eddy viscosity $v$ to be proportional to $S$ :

$$
v=l^{2} S,
$$

where the length scale of mixing of turbulent eddies

$$
l=\eta \sqrt{c_{t}},
$$

and $c_{t}$ is an empirical constant related to the strength of the mixing. Relation (6) is valid some distance from boundaries. The resulting relation for the turbulent mean stress (5) is

$$
\tau_{i j}=2 c_{t} \eta^{2} S S_{i j}=c_{t} \eta^{2} S\left(\frac{\partial u_{i}}{\partial x_{j}}+\frac{\partial u_{j}}{\partial x_{i}}\right),
$$

and together with (3) and (4) it governs the dynamical evolution of the flow. The primary quantities we want to determine are the fluid thickness $\eta$ and the mean velocity field $\vec{u}$.

Boundary conditions We set boundary conditions on the mean bed $(z=$ 0 ) and on the free surface $(z=\eta)$. The mean bed stops vertical mean flow so that

$$
w=0 \quad \text { and } \quad S=\frac{1}{\sqrt{2}} \frac{\partial u}{\partial z} \quad \text { on } z=0,
$$

but allows slip to account for a relatively thin turbulent boundary layer,

$$
u=c_{u} \eta \frac{\partial u}{\partial z} \quad \text { on } z=0 .
$$


The physical meaning of (10) is that the mean bed $z=0$ is located at the lower edge of the turbulent boundary layer where there is no mean flow in the vertical direction, but the mean flow in the lateral direction is proportional to the friction velocity $\mathfrak{u}_{*}$,

$$
u \propto u_{*} \quad \text { on } z=0
$$

where $\mathfrak{u}_{*} \equiv \sqrt{\left.\mu \frac{\partial u}{\partial z}\right|_{z=0}}$, and $\mu$ is the molecular viscosity. At $z=0$ the viscous and turbulent effects are assumed approximately equal, therefore $\left.\mu \approx v\right|_{z=0}$ and the friction velocity is defined as $u_{*}=\sqrt{\left.\left.v\right|_{z=0} \frac{\partial u}{\partial z}\right|_{z=0}}$. Taking into account (9), (6) and (7) it follows that the value of the constant $\boldsymbol{c}_{\mathfrak{u}}$ in (10) depends on the proportionality (11). This value has to be determined empirically, similarly to $c_{\mathfrak{t}}$ : the parameter $c_{\mathfrak{u}} \eta$ in (10) characterises the 'slip length' on the bed.

At $z=\eta$ we have the mean free surface. The kinematic condition there is

$$
\frac{\partial \eta}{\partial t}+u \frac{\partial \eta}{\partial x}=w \quad \text { on } z=\eta
$$

Since the density of the air is very low, we assume constant pressure of the air on the fluid surface, which in our system of units is set to zero without loss of generality. Thus the turbulent mean stress normal to the free surface is also zero,

$$
-p+\frac{1}{1+\eta_{x}^{2}}\left[\tau_{33}-2 \frac{\partial \eta}{\partial x} \tau_{13}+\left(\frac{\partial \eta}{\partial x}\right)^{2} \tau_{11}\right]=0 \quad \text { on } z=\eta .
$$

Lastly, there must be no turbulent mean tangential stress at the free surface

$$
\left[1-\left(\frac{\partial \eta}{\partial x}\right)^{2}\right] \tau_{13}+\frac{\partial \eta}{\partial x}\left(\tau_{33}-\tau_{11}\right)=0 \quad \text { on } z=\eta .
$$




\section{Centre manifold technique}

We proceed to solve the governing equations of the flow (3), (4) and (8) by applying a new approach based on centre manifold theory. As previously mentioned, our primary interest is the time evolution of the height of the flow $\eta(x, t)$ and depth averaged lateral velocity $\bar{u}(x, t)=\frac{1}{\eta} \int_{0}^{\eta} u d z$. However, we do not assume a prescribed empirical vertical structure $\mathfrak{u}(z)$, instead we use centre manifold theory to resolve turbulent dynamics across the fluid layer by separating the influence of the terms involved in (3), (4) and (8) on the basis of their time scales. In such a way the vertical turbulent mixing and the preservation of mass are recognized as dominant dynamics, and the lateral derivatives $\partial_{x}^{n} \equiv\left[\frac{\partial^{n} \bar{u}}{\partial x^{n}}, \frac{\partial^{n} \eta}{\partial x^{n}}\right]$, the gravitational forcing $\vec{g}$ and the curvature of the free surface, are recognized as imposed perturbations that generate slow, long term, large scale evolution.

To implement this approach we provide a special mechanism in (3), (4) and (8) and/or in the boundary conditions, such that these equations are solved by a family of shear flows of constant thickness. On this basis two neutral modes of the dynamics of the flow are defined, and the space of equilibria of (3)-(4) and (8) then parameterized using $\overline{\mathfrak{u}}(x)$ and $\eta(x)$. To allow this we add an artificial tangential stress on the free surface, later removed, and proportional to the square of the local velocity $\mathfrak{u}^{2}(\eta)$. The boundary condition (14) is replaced by

$$
\left[1-\left(\frac{\partial \eta}{\partial x}\right)^{2}\right] \tau_{13}+\frac{\partial \eta}{\partial x}\left(\tau_{33}-\tau_{11}\right)=\frac{(1-\gamma) c_{t}}{\sqrt{2}\left(1+c_{u}\right)^{2}} u^{2} \quad \text { on } z=\eta
$$

where $\gamma$ is an artificial parameter ranging from zero to one. When we later set $\gamma=1$ the physical boundary condition (14) is recovered. However, when $\gamma=0$ and $g_{1}=\partial_{x}=0$, then from (5) $\tau_{13}=v \partial u / \partial z$ and, hence, the artificial free surface condition (15) reduces to

$$
u=\left(1+c_{u}\right) \eta \frac{\partial u}{\partial z} \quad \text { on } z=\eta
$$


As a result, when $\gamma=0$ then (10) and (16) create the neutral mode of artificial shear flow $\mathfrak{u} \propto \boldsymbol{c}_{\mathfrak{u}}+\zeta$, where $\zeta$ is the scaled normal coordinate, namely

$$
\mathrm{u}(x, z, \mathrm{t})=\overline{\mathrm{u}}(x, \mathrm{t}) \frac{\mathrm{c}_{\mathfrak{u}}+\zeta}{\mathrm{c}_{\mathfrak{u}}+\frac{1}{2}}, \quad \zeta \equiv z / \eta .
$$

In such a way the parameter $\gamma$ provides a transition between the boundary condition (16), which establishes the 'slow' centre manifold, and the boundary condition (14), which is physically reasonable. We base our analytic analysis on the case $\gamma=0$, construct power series solutions in $\gamma$, and seek results for the physical case $\gamma=1$. Centre manifold theory $[1,7$, e.g.] assures us that the slow manifold exists and emerges in some finite domain around $\gamma=0$. Since we anticipate that the transition from $\gamma=0$ to $\gamma=1$ is smooth, we consequently expect that in the physical case of $\gamma=1$ the solutions of (3)-(4) and (8) are still in the finite domain of validity of the centre manifold theorems. We previously verified the validity of the slow manifold by comparing with analytic solutions [3], and we applied it to derive the vertical structure of turbulence for flood flows [2].

Relation (17) defines one neutral mode of the dynamics. Conservation of fluid provides the second neutral mode: when $\gamma=g_{1}=\partial_{x}=0$ then $\eta=$ constant is an equilibrium. Thus when $\gamma=g_{1}=\partial_{x}=0$, there is a two parameter subspace of equilibria corresponding to uniform shear flow, $\mathfrak{u} \propto \boldsymbol{c}_{\mathfrak{u}}+\zeta$, of any constant thickness $\eta$. For large enough lateral length scales, these equilibria occur independently at each location $x$ [11] and hence the space of equilibria are in effect parameterized by $\bar{u}(x)$ and $\eta(x)$. Provided we treat lateral derivatives $\partial_{x}$ as a modifying influence, that is, provided solutions vary slowly enough in $x$, centre manifold theorems $[1,7$, e.g.] assure us that the evolution of the system (3), (4) and (8) develops on a slow manifold $\mathcal{M}$ with the following properties:

- $\mathcal{M}$ is a perturbation of the subspace of equilibria;

- $\mathcal{M}$ exists for a finite range of parameters $\partial_{x}, \gamma$ and $g_{1}$;

- $\mathcal{M}$ is parameterized by the average $\bar{u}(x, t)$ and $\eta(x, t)$, therefore the 
evolution on $\mathcal{M}$ is low dimensional;

- $\mathcal{M}$ attracts solutions from all nearby initial conditions and hence emerges exponentially quickly;

- $\mathcal{M}$ may be approximated by power series in $\partial_{x}, \gamma$ and $g_{1}$ to the same order of error as the governing PDEs are satisfied.

Roberts [12] presented details of the algorithm to construct the slow manifold $\mathcal{M}$ using asymptotic series and computer algebra. The cross fluid structure of the velocity, pressure and strain rate forms the slow manifold, which is defined for flow with no lateral variations $\partial_{x}=0$. An approximation for the lateral velocity shear profile is

$$
\begin{aligned}
u= & \bar{u} \frac{c_{u}+\zeta}{c_{u}+\frac{1}{2}}+\gamma \bar{u} \frac{\left(1+c_{u}\right)\left[\left(1+4 c_{u}\right)\left(c_{u}+\zeta\right)-2\left(1+2 c_{\mathfrak{u}}\right)\left(3 c_{u} \zeta^{2}+\zeta^{3}\right)\right]}{4\left(1+2 c_{\mathfrak{u}}\right)^{2}\left(1+3 c_{u}+3 c_{u}^{2}\right)} \\
& +\frac{g_{1} \eta}{\bar{u}} \frac{\left[\left(5+6 c_{\mathfrak{u}}\right)\left(c_{\mathfrak{u}}+\zeta\right)-6\left(2+7 c_{\mathfrak{u}}+6 c_{\mathfrak{u}}^{2}\right) \zeta^{2}+6\left(1+2 c_{\mathfrak{u}}\right)^{2} \zeta^{3}\right]}{48 \sqrt{2} c_{\mathfrak{t}}\left(1+3 c_{\mathfrak{u}}+3 c_{\mathfrak{u}}^{2}\right)} \\
& +\mathcal{O}\left(\gamma^{2}+g_{1}^{2}+\partial_{x}\right),
\end{aligned}
$$

and the magnitude of the rate of strain tensor is

$$
\begin{aligned}
S= & \frac{\bar{u}}{\eta} \frac{\sqrt{2}}{1+2 c_{u}}+\frac{\gamma \bar{u}}{\eta} \frac{\sqrt{2}\left(1+c_{u}\right)\left[\left(1+4 c_{u}\right)-6\left(1+2 c_{u}\right)\left(2 c_{u} \zeta+\zeta^{2}\right)\right]}{8\left(1+2 c_{u}\right)^{2}\left(1+3 c_{u}+3 c_{u}^{2}\right)} \\
& +\frac{g_{1}}{\bar{u}} \frac{\left[\left(5+6 c_{u}\right)-12\left(2+7 c_{u}\right) \zeta+18\left(1+2 c_{u}\right)^{2} \zeta^{2}\right]}{96 c_{t}\left(1+3 c_{u}+3 c_{u}^{2}\right)} \\
& +\mathcal{O}\left(\gamma^{2}+g_{1}^{2}+\partial_{x}\right),
\end{aligned}
$$

for flow with hydrostatic pressure $p=g_{3} \eta(1-\zeta)$. The corresponding evolution of the mean lateral velocity is

$$
\begin{aligned}
\frac{d \bar{u}}{d t}= & -\frac{\sqrt{2} 3 c_{t}\left(1+c_{u}\right)}{\left(1+2 c_{u}\right)\left(1+3 c_{u}+3 c_{u}^{2}\right)} \frac{\gamma \bar{u}^{2}}{\eta}+\frac{\frac{3}{4}+3 c_{u}+3 c_{u}^{2}}{1+3 c_{u}+3 c_{u}^{2}} g_{1} \\
& +\mathcal{O}\left(\gamma^{2}+g_{1}^{2}+\partial_{x}\right) .
\end{aligned}
$$


To obtain a physical model from (18)-(20) we set the artificial parameter $\gamma=1$ which enforces the physical free surface boundary condition (14). Then (18)-(20) describe the equilibrium of an uniform open channel flow; for example, the second term in (20) accelerates the flow by the lateral gravitational forcing, until it is balanced by turbulent drag on the bed, the first term. Higher order models account for more physical processes.

\section{Calibration}

We construct the slow manifold for small lateral derivatives, small lateral forcing and small perturbation of the free surface condition. Later we analyze the time evolution of $\eta(x, t)$ and $\bar{u}(x, t)$ to verify that these criteria are satisfied for specific flows of interest. Also the Smagorinsky 'mixing' coefficient $c_{t}$ and bed 'slip' coefficient $c_{\mathfrak{u}}$ have to be set to appropriate values, because (18)-(20) with $\gamma=1$ define a family of equilibrium flows. When $c_{t}$ and $c_{\mathfrak{u}}$ are set, equations (18)-(20) with $\gamma=1$ will describe the lateral velocity shear profile and equilibrium of a specific uniform flow with fully developed turbulence, since these relations were derived assuming no lateral variations $\partial_{x}=0$.

The stationary channel flow is closest to these assumptions, it is well studied, and there are experimental data showing the structure of the turbulence in the interior of the flow. Summarizing many experiments, Nezu [9] gave empirical formulae for the structure of the turbulent energy $k(z)=$ $4.78 u_{*}^{2} \exp (-2 \zeta)$ and dissipation $\epsilon(z)=9.8 u_{*}^{3} \exp (-3 \zeta) /(\eta \sqrt{\zeta})$; from these the profile of the eddy viscosity $v(z)=C_{\mu} k^{2} / \epsilon$, thus the depth averaged eddy viscosity is $\bar{v}=0.0796 \eta u_{*}$. From (18)-(20) these depth averaged flow parameters can be estimated; for example, the mean velocity is

$$
\bar{u}=\frac{1}{2}\left[\frac{\left(1+2 c_{u}\right)^{3}}{c_{t} \sqrt{2}\left(1+c_{u}\right)}\right]^{1 / 2} \sqrt{g_{1} \eta} .
$$


By choosing $c_{t}=1 / 50$ and $c_{\mathfrak{u}}=11 / 6$ this equilibrium velocity and the average eddy viscosity correspond to the empirical results [9], taking into account that $\mathfrak{u}_{*}=\sqrt{g_{1} \eta}$ for stationary channel flow driven only by the force of gravity.

Our next step is to estimate the spatio-temporal dynamics of non-stationary, out-of-equilibrium, channel flow. In our model they are described by the time evolution of the flow height $\eta(x, t)$ and the depth averaged lateral velocity $\bar{u}(x, t)$. They come as a result of taking into account the relatively slow variations in the lateral direction $x$, and small but non-zero $\partial_{x}$, therefore these functions are smooth and slow in $x$. Georgiev et al. [3] describe more details of the slow dynamics. The long time, large scale, evolution of $\eta(x, t)$ and $\bar{u}(x, t)$ are described by the conservation equation

$$
\frac{\partial \eta}{\partial t}+\frac{\partial}{\partial x}(\eta \bar{u})=0
$$

and the momentum equation

$$
\begin{aligned}
\frac{\partial \bar{u}}{\partial t}= & -1.045 \bar{u} \frac{\partial \bar{u}}{\partial x}-0.00311 \gamma|\bar{u}| \bar{u} / \eta+0.985\left(g_{1}-g_{3} \frac{\partial \eta}{\partial x}\right) \\
& +0.058 \eta \frac{\partial|\bar{u}|}{\partial x} \frac{\partial \bar{u}}{\partial x}+0.259 \eta|\bar{u}| \frac{\partial^{2} \bar{u}}{\partial x^{2}}+0.522|\bar{u}| \frac{\partial \eta}{\partial x} \frac{\partial \bar{u}}{\partial x} \\
& -0.015 \bar{u}^{2} \frac{\partial \eta}{\partial x} / \eta-0.007|\bar{u}| \bar{u}\left(\frac{\partial \eta}{\partial x}\right)^{2} / \eta-0.007|\bar{u}| \bar{u} \frac{\partial^{2} \eta}{\partial x^{2}} \\
& +O\left(\gamma^{3 / 2}+g_{1}^{3 / 2}+g_{3}^{3}+\partial_{x}^{3}\right) .
\end{aligned}
$$

Equation (23) contains the inertial term $\bar{u}_{t}$, self-advection term $\bar{u} \frac{\partial \bar{u}}{\partial x}$, bed drag $|\bar{u}| \bar{u} / \eta$, gravitational forcing $g_{1}-g_{3} \frac{\partial \eta}{\partial x}$, and other terms related to turbulent mixing in the lateral direction. We emphasize that the coefficients at these terms are supported by centre manifold theory instead of using empirical relations, such as Chezy law for bed drag [4]. Remarkably, in our model (23) the coefficient of gravitational forcing lessens the effect of gravity by $1-2 \%$ (reports confirm such a decrease [13]). 


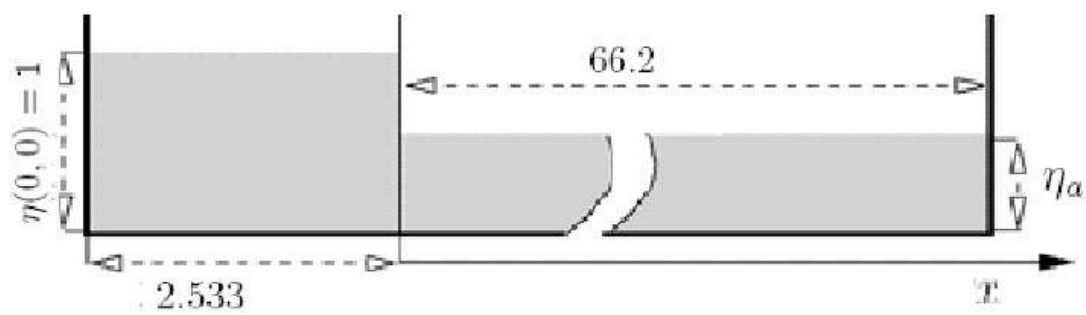

FiguRE 1: Schematic arrangement and geometric dimensions of the dambreak experiments of Janosi et al. [5].

Applying (22)-(23) to dam-break flow, it is interesting to estimate the relative role of these terms in the evolution of $\eta(x, t)$. In doing so we use the experimental results of Janosi et al. [5] on dam-break flow. Figure 1 shows the experimental setting. The initial filling height is assumed $\eta(0,0)=1$ in our system of units, $g_{3}=1$. The lock begins to open at $t=0$ and is fully opened at $t=0.808$. We focus on two experiments with $R e \approx 30,000$ 40,000 , ambient height of the flow being $\eta_{a}=0.12$ and $\eta_{a}=0.253$. During these laboratory experiments various phenomena related to intensive turbulent mixing were observed: a developed propagating bore, air bubbles, surface wave breaking in forward and backward directions, and an unstable mushroom-like surface wave, which was also reported by Stansby et al. [14].

During the experiments, snapshots were taken of the water height $\eta(x, t)$ at successive moments of time [5]. We choose to compare $\eta(x, t)$ from our modelling with the experimental $\eta(x, t)$, rather than compare $\bar{u}(x, t)$, since the observations of the height of the flow are more precise than the measurements of the mean velocity. Equations (22) and (23) are solved numerically using EPDCOL solver of PDEs created by Keast and Muir [6], which is a successor of the PDECOL solver of Madsen and Sincovec [8]; it employs a collocation based method-of-lines approach. Figure 2 compares the height of the flow for the most distant moment of time. To estimate the relative role of the terms in (23), the profile of $\eta$ is calculated with some of the terms omitted (except for the gravitational forcing and $\eta|\bar{u}| \frac{\partial^{2} \bar{u}}{\partial x^{2}}$ ), and compared with the 


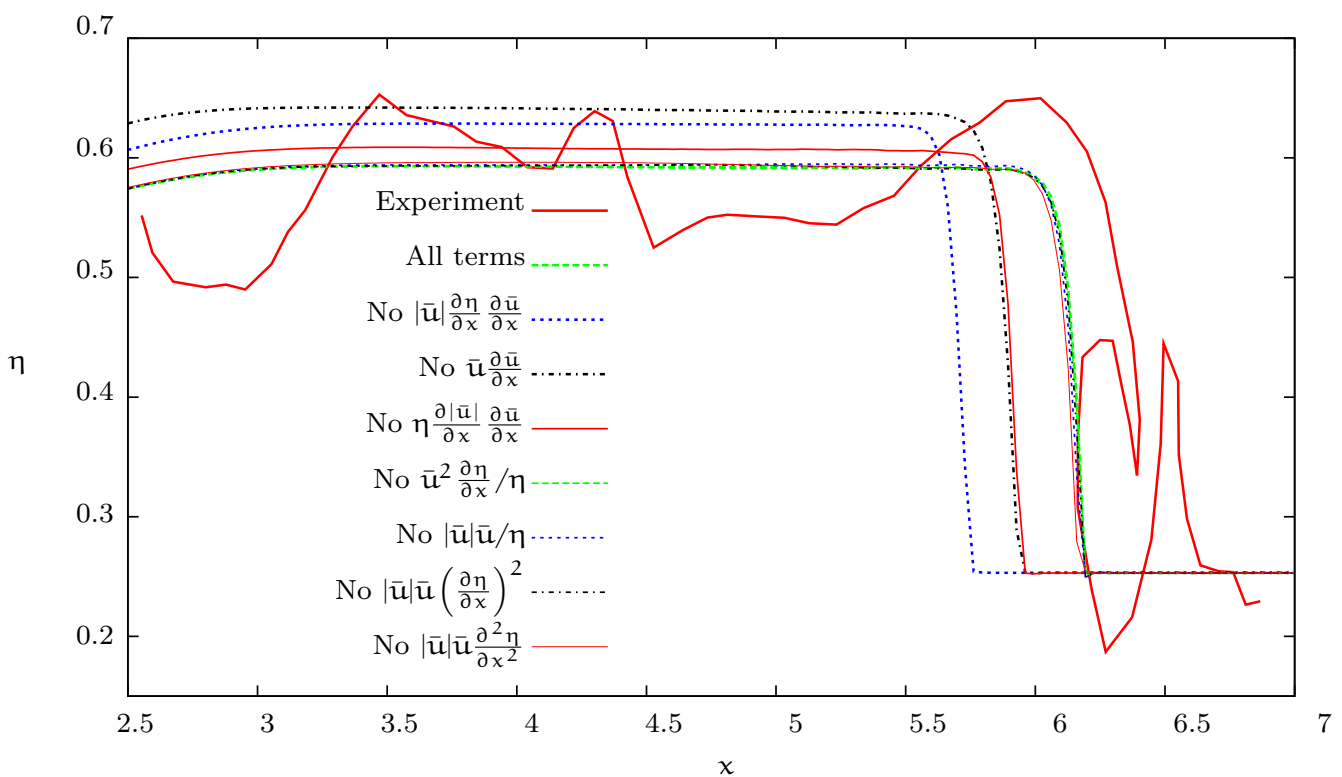

Figure 2: Comparison of experimental and computed $\eta$ for $\eta_{a}=0.253$ and $\mathrm{t}=4.794$. 
solution produced using all the terms of (23). We found that apart from the self-advection term $\bar{u} \frac{\partial \bar{u}}{\partial x}$, only the two terms $|\bar{u}| \frac{\partial \eta}{\partial x} \frac{\partial \bar{u}}{\partial x}$ and $\eta \frac{\partial|\bar{u}|}{\partial x} \frac{\partial \bar{u}}{\partial x}$ have a significant effect on the lateral speed of the water front, these terms are related to turbulent mixing in lateral direction. Thus, the terms representing turbulent mixing in the third line of (23) appear not significant, and for practical purposes the model (23) may be simplified to

$$
\begin{aligned}
\frac{\partial \bar{u}}{\partial t} \approx & -1.045 \bar{u} \frac{\partial \bar{u}}{\partial x}-0.00311|\bar{u}| \bar{u} / \eta+0.985\left(g_{1}-g_{3} \frac{\partial \eta}{\partial x}\right) \\
+ & 0.058 \eta \frac{\partial|\bar{u}|}{\partial x} \frac{\partial \bar{u}}{\partial x}+0.259 \eta|\bar{u}| \frac{\partial^{2} \bar{u}}{\partial x^{2}}+0.522|\bar{u}| \frac{\partial \eta}{\partial x} \frac{\partial \bar{u}}{\partial x} .
\end{aligned}
$$

Equation (24), and the simulations, use $\gamma=1$ to restore the physical boundary condition (14) on the free surface. Equation (24) can be simplified further by approximating the coefficients to two decimals and combining the last three terms into one

$$
\begin{aligned}
\frac{\partial \bar{u}}{\partial t}+1.05 \bar{u} \frac{\partial \bar{u}}{\partial x} \approx & -0.0031|\bar{u}| \bar{u} / \eta+0.98\left(g_{1}-g_{3} \frac{\partial \eta}{\partial x}\right) \\
& +0.26 \frac{|\bar{u}|^{0.78}}{\eta} \frac{\partial}{\partial x}\left(\eta^{2}|\bar{u}|^{0.22} \frac{\partial \bar{u}}{\partial x}\right) .
\end{aligned}
$$

This equation accumulates the influence of the factors major factors: advection, bed drag, gravitational forcing and lateral turbulent mixing. We re-iterate that the models (24) and (25) are not derived by depth averaging, but by using centre manifold theory, which systematically accounts for interactions of vertical profiles of the velocity/stress and bed drag with lateral space variations. This approach provides a solid ground for regularizing the dissipation in the second lines of (24) and (25).

\section{$5 \quad$ Dam-break modelling}

Figures 3 and 4 compare computed profiles of the dam-break flow for ambient heights $\eta_{a}=0.12$ and $\eta_{a}=0.253$ with snapshots from the experiments. 

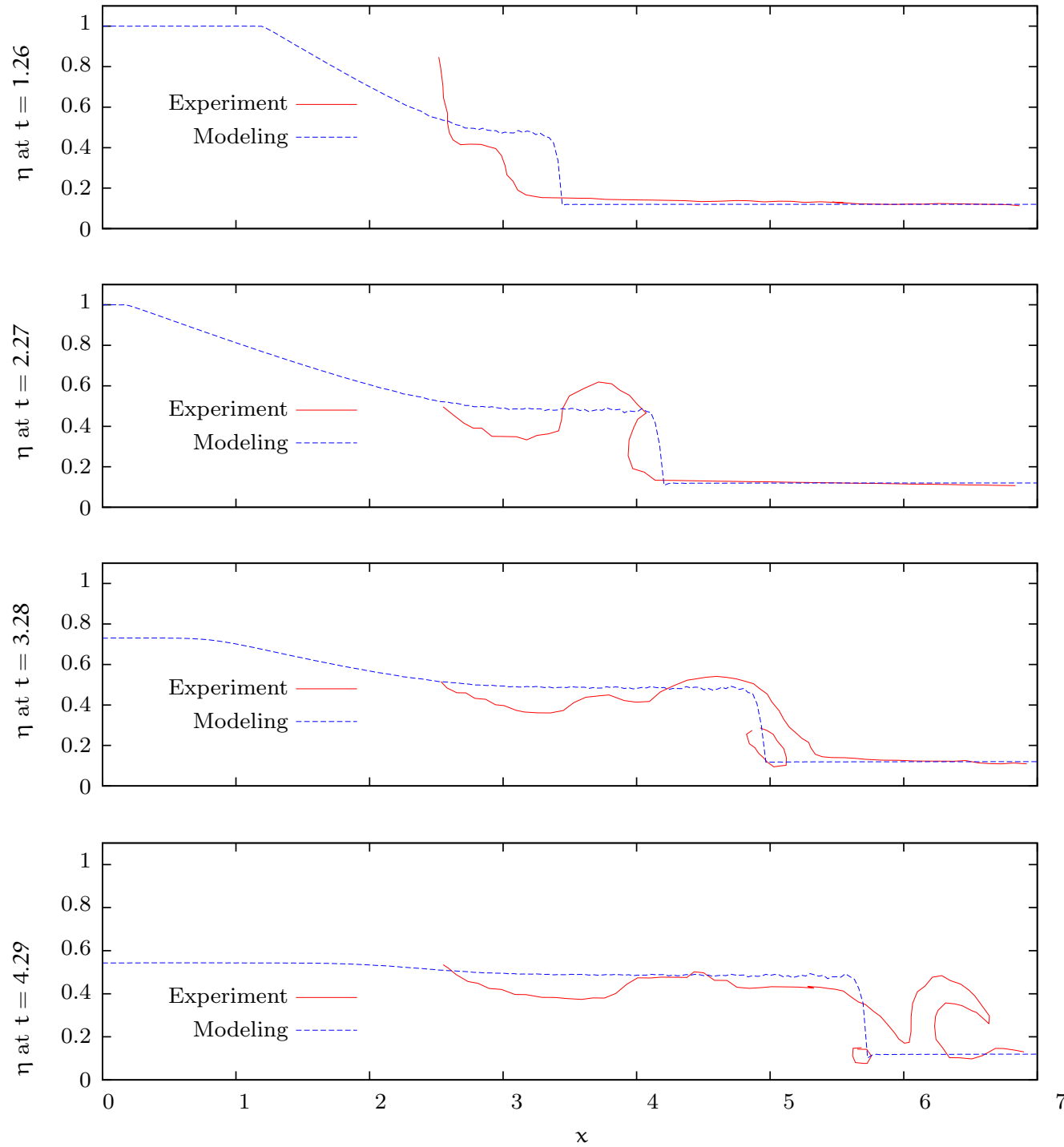

FiguRE 3: Comparison of experimental and computed $\eta$ for $\eta_{a}=0.12$. 

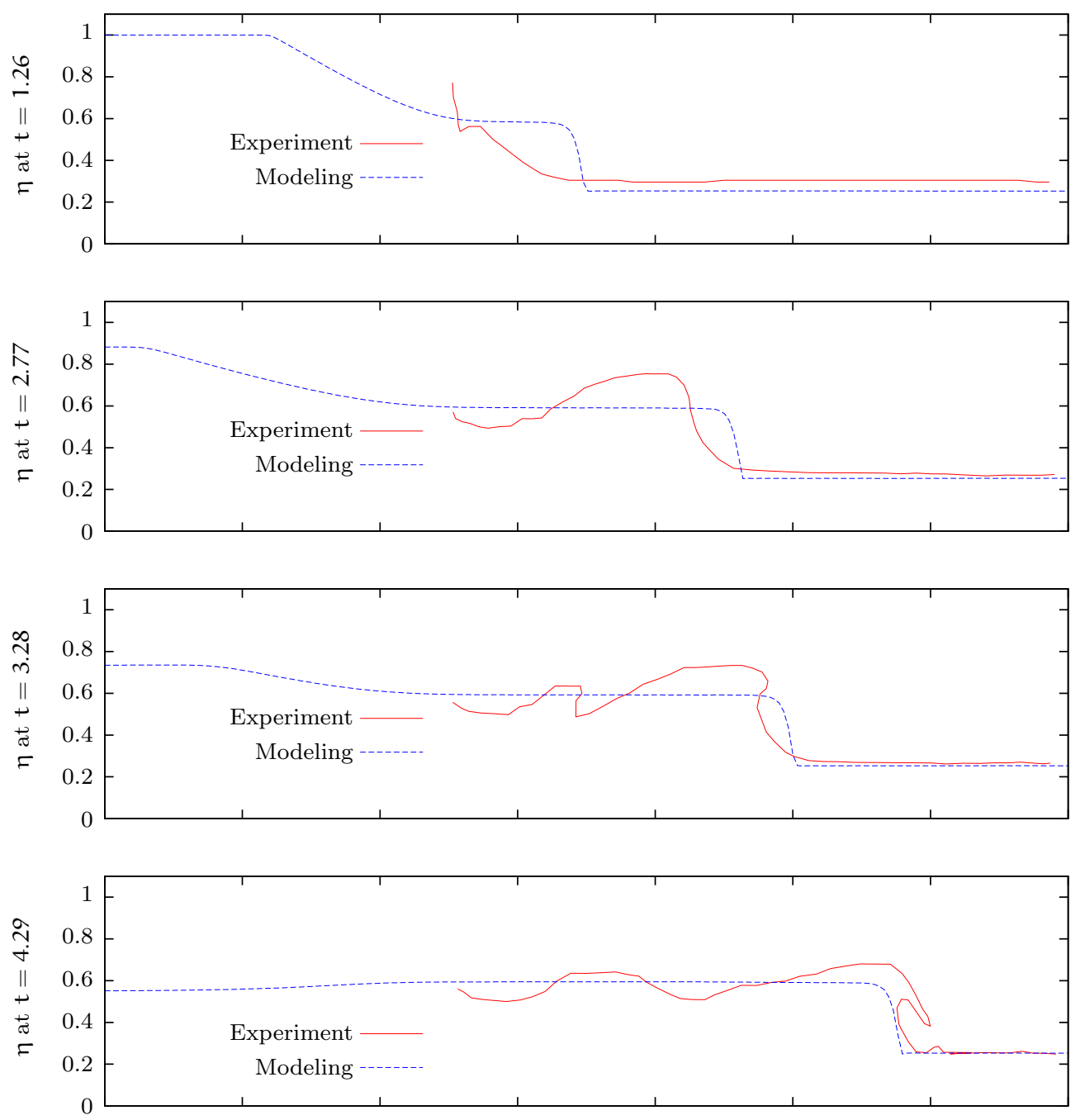

Figure 4: Comparison of experimental and computed $\eta$ for $\eta_{a}=0.253$. 
When $\eta_{a}=0.12$ the static layer at the bottom is thinner and its interaction with the propagating bore causes wave breaking and entrainment of air bubbles. In our modelling we assume that the lock was fully opened at $t=0$, and, as a consequence, the computed front of the flow is ahead of the experimental one at early moments of time; later the speed of the two fronts become close. Note that the experimental snapshots show instantaneous height of the flow, while we computed the ensemble average height. Therefore we do not expect to reproduce fine details of the transient turbulent structures, such as mushroom-like surface wave, wave breaking and air bubbles. They are highly curved and therefore are far from being small perturbations of the free surface. Nevertheless, not far behind the propagating bore the profile of the surface is smoother and its ensemble average is close to the computed profile.

When the static layer at the bottom is thick $\left(\eta_{a}=0.253\right)$, its interaction with the propagating bore is delayed and so is the wave breaking in the forward direction. There are no captured air bubbles, and the mushroomlike surface wave is larger and smoother. As a result, the correspondence with the computed profile is better and the computed front is closer to the propagating bore. In the backward direction the computed profile is close to the ensemble averaged experimental profile.

\section{Conclusion}

The presented approach using centre manifold techniques can reliably describe the relatively slow, long term, large scale, modes of turbulent dambreak flow. Instead of assuming empirical profiles or depth-averaging the equations of the flow, we resolve turbulent dynamics across the fluid layer and calibrate the resulted family of solutions. The resulted relations (22) and (25) evaluate the interactions of vertical profiles of the velocity/stress and bed drag with lateral space variations. 


\section{References}

[1] J. Carr. Applications of centre manifold theory, volume 35 of Applied Mathematical Sciences. Springer-Verlag, 1981. C1039

[2] D. J. Georgiev, A. J. Roberts, and D. V. Strunin. The dynamics of the vertical structure of turbulence in flood flows. ANZIAM Journal, 48(CTAC-2006):C573-C590, 2007. http://anziamj . austms.org.au/ ojs/index.php/ANZIAMJ/article/view/124. C1039

[3] D. J. Georgiev, A. J. Roberts, and D. V. Strunin. Nonlinear dynamics on centre manifolds describing turbulent floods: k- $\omega$ model. Discrete and Continuous Dynamical Systems Supplements, (Special):419-428, 2007. http:

//aimsciences . org/journals/pdfs . jsp?paperID=2848\&mode=full. C1039, C1042

[4] A. J. Hogg and D. Pritchard. The effects of hydraulic resistance on dam-break and other shallow inertial flows. Journal of Fluid Mechanics, 501:179-212, 2004. C1034, C1042

[5] I. M. Janosi, D. Jan, K. G. Szabo, and T. Tel. Turbulent drag reduction in dam-break flows. Experiments in Fluids, 37:219-229, 2004. C1034, C1043

[6] P. Keast and P. H. Muir. EPDCOL: A more efficient PDECOL code. ACM Transactions on Mathematical Software, 17(2):153-166, 1991. $\mathrm{C} 1043$

[7] Y. A. Kuznetsov. Elements of applied bifurcation theory, volume 112 of Applied Mathematical Sciences. Springer-Verlag, 1995. C1039

[8] N. K. Madsen and R. F. Sincovec. PDECOL: General collocation software for partial differential equations. ACM Transactions on Mathematical Software, 5(3):326-351, 1979. C1043 
[9] I. Nezu. Open-channel flow turbulence and its research prospects in the 21st century. Journal of Hydraulic Engineering, 131(4):229-246, April 2005. C1041, C1042

[10] T. M. Özgökmen, T. Iliescu, P. F. Fischer, A. Srinivasan, and J. Duan. Large eddy simulation of stratified mixing and two-dimensional dam-break problem in a rectagular enclosed domain. Ocean Modelling, 16:106-140, 2007. C1034, C1036

[11] A. J. Roberts. Low-dimensional modelling of dynamics via computer algebra. Computer Physics Communications, 100:215-230, 1997. C1039

[12] A. J. Roberts. Computer algebra describes flow of turbulent floods via the Smagorinsky large eddy closure. Technical report, University of Southern Queensland, 2008. http://eprints.usq. edu.au/4008/. C1040

[13] A. J. Roberts, D. J. Georgiev, and D. V. Strunin. Model turbulent floods with the Smagorinski large eddy closure.

http://arxiv.org/abs/0805.3192/. C1034, C1042

[14] P. K. Stansby, A. Chegini, and T. C. D. Barnes. The initial stages of dam-break flow. Journal of Fluid Mechanics, 374:407-424, 1998. C1034, C1043

\section{Author addresses}

1. D. J. Georgiev, Commonwealth Scientific and Industrial Research Organisation, Exploration and Mining, P.O. Box 1130, Bentley, Western Australia 6102, Australia. mailto:Dian.Georgiev@csiro.au

2. A. J. Roberts, School of Mathematical Sciences, University of Adelaide, South Australia 5005, Australia. 
mailto: anthony.roberts@adelaide.edu.au

3. D. V. Strunin, Computational Engineering and Sciences Research Centre, University of Southern Queensland, Toowoomba, Queensland 4352, Australia. mailto: strunin@usq. edu. au 